\title{
Evaluating disease-modifying effects of a sigma-1 receptor agonist in an animal model of ALS
}

\section{Héctor Estévez-Silva ${ }^{1}, 2$, Tomás Mediavilla ${ }^{1}$, Bruno Lima-Giacobbo ${ }^{1}$, Fahad Sultan ${ }^{1}$, Daniel Marcellino ${ }^{1}$}

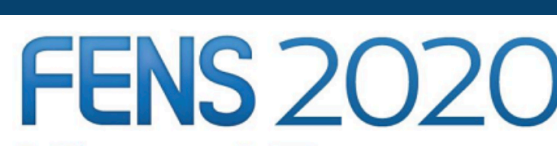

Virtual Forum

11-15 July 2020

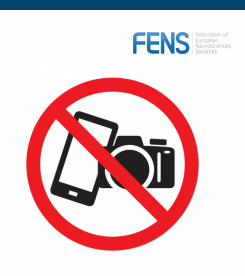

${ }^{1}$ Department of Integrative Medical Biology, Umeå University, Umeå, Sweden.

2 Instituto de Tecnologías Biomédicas (ITB), Departamento de Ciencias Médicas Básicas, Facultad de Ciencias de la Salud, Universidad de La Laguna, Tenerife. Spain.

\section{INTRODUCTION}

Mutations in superoxide dismutase 1 (SOD1) are related to familial amyotrophic lateral sclerosis (fALS), producing degeneration of motor neurons. Transgenic mice carrying the human SOD1G93A mutation exhibit cachexia, paralysis, and early death due to SOD1 protein misfolding and aggregation. Recent research has indicated that sigma-1 receptors (S1R), in which mutations are linked to juvenile ALS, prevents aberrant protein conformation by acting as a molecular chaperone when activated by specific agonists such as pridopidine.

\section{AlM}

We evaluated the putative disease-modifying effects of pridopidine in a delayed onset hSOD1G93A mouse model $\left(G 93 A^{*}\right)$, initiating treatment prior to the development of symptoms and its subsequent withdrawal after 4 weeks.

\section{EXPERIMENTAL DESIGN}

Animals. All experiments were performed using hemizygous hSOD $1{ }^{\text {G93A }}$ mice with a $20 \%$ loss of transgene $\left(G 93 A^{*}\right)$ and their nontransgenic (nTg) littermates. Similar copy numbers were determined in all animals used in the study (qPCR).

Treatment. At 18 weeks, osmotic pumps were implanted (s.c.) to provide a continuous release of pridopidine $(3.0 \mathrm{mg} / \mathrm{kg} / \mathrm{day})$ or vehicle (saline) for 4 weeks before its withdrawal.

Behavioral tests. The Inverted Screen test (I.S.T), Pole test (P.T), and an analysis of gait using the Footprint test (G.T) were performed to evaluate motor coordination as indicated below:

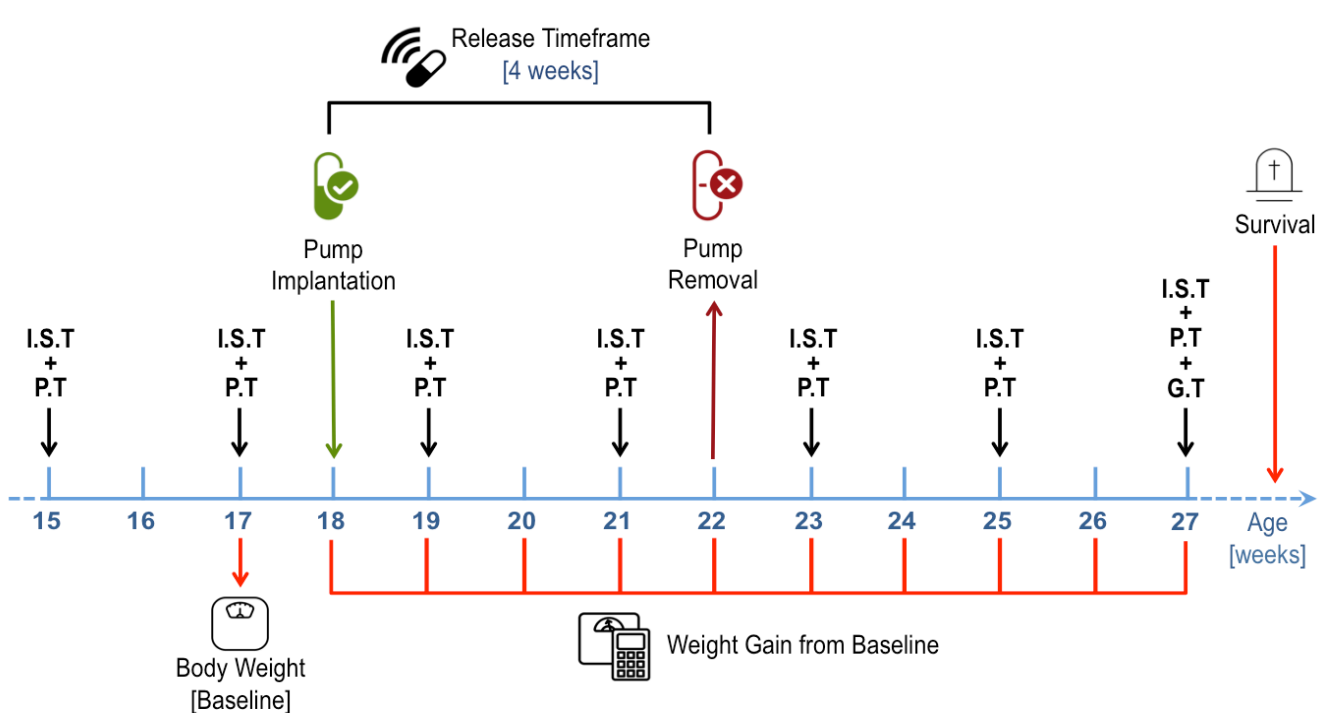

Statistics. Data were analyzed by 2-way ANOVA followed by Bonferroni's multiple comparisons test. Gait Test was analyzed using 1-way ANOVA and Holm-Sidak multiple comparisons test.

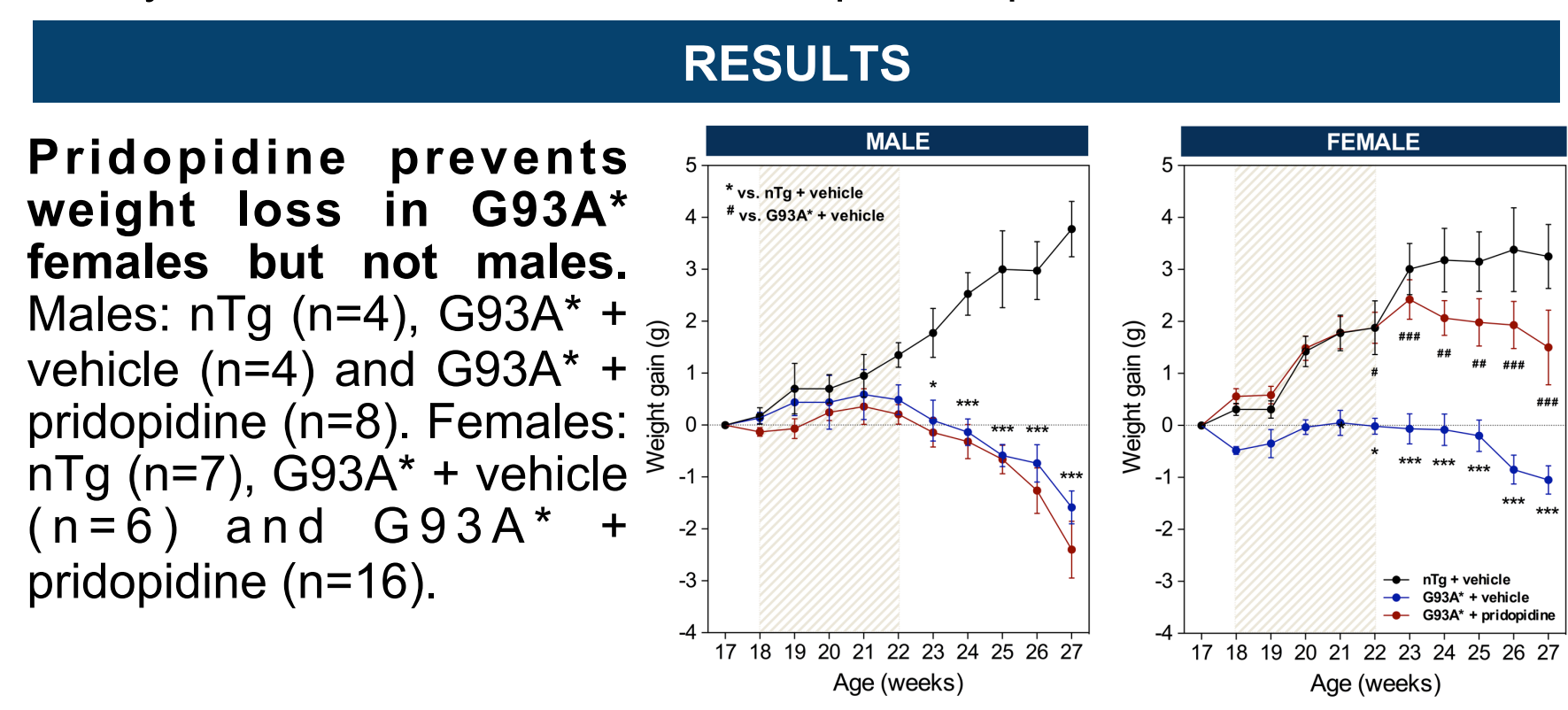

Pridopidine-treatment extends the lifespan of $\mathrm{G} 9 \mathrm{~A}^{*}$ mice. Pridopidinetreated group (32.9 weeks) vs. salinetreated (30.4 weeks). Kaplan-Meier survival plot. Statistical analysis by Gehan-Breslow-Wilcoxon test indicates a $p$-value of 0.0637 . Mice were euthanized when they unable to right themselves within $5 \mathrm{~s}$ after being placed on their side.
Differences in body weight influence performance of the inverted screen test. G93A* mice exhibit a progressive loss in grip strength, a phenotype that occurs more rapidly in males than females. In females, pridopidine-treated mice exhibited a reduced latency to fall than saline-treated mice, likely as a result of increased weight. ${ }^{*} p<0.05$ and ${ }^{* * *} p<0.001$ in $\mathrm{nTg}$ littermates vs. G93A* ${ }^{*} \mathrm{p}<0.001$ in saline-treated $v s$. pridopidine-treated $G 93 A^{*}$ mice. Males: $n T g(n=4), \quad G 93 A^{*}+$ vehicle $(n=4)$ and $G^{2} 3 A^{*}+$ pridopidine $(n=5)$. Females: $n T g(n=7), \quad G 93 A^{*}+$ vehicle $(n=6)$ and $G 93 A^{*}+$ pridopidine $(n=9)$.

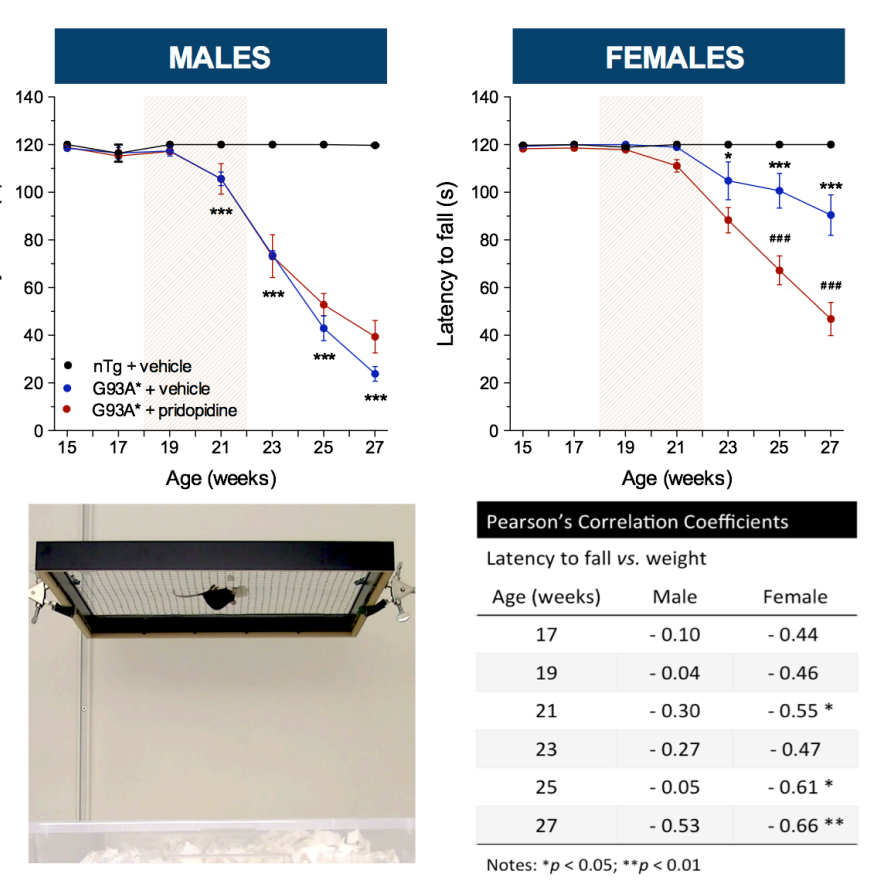

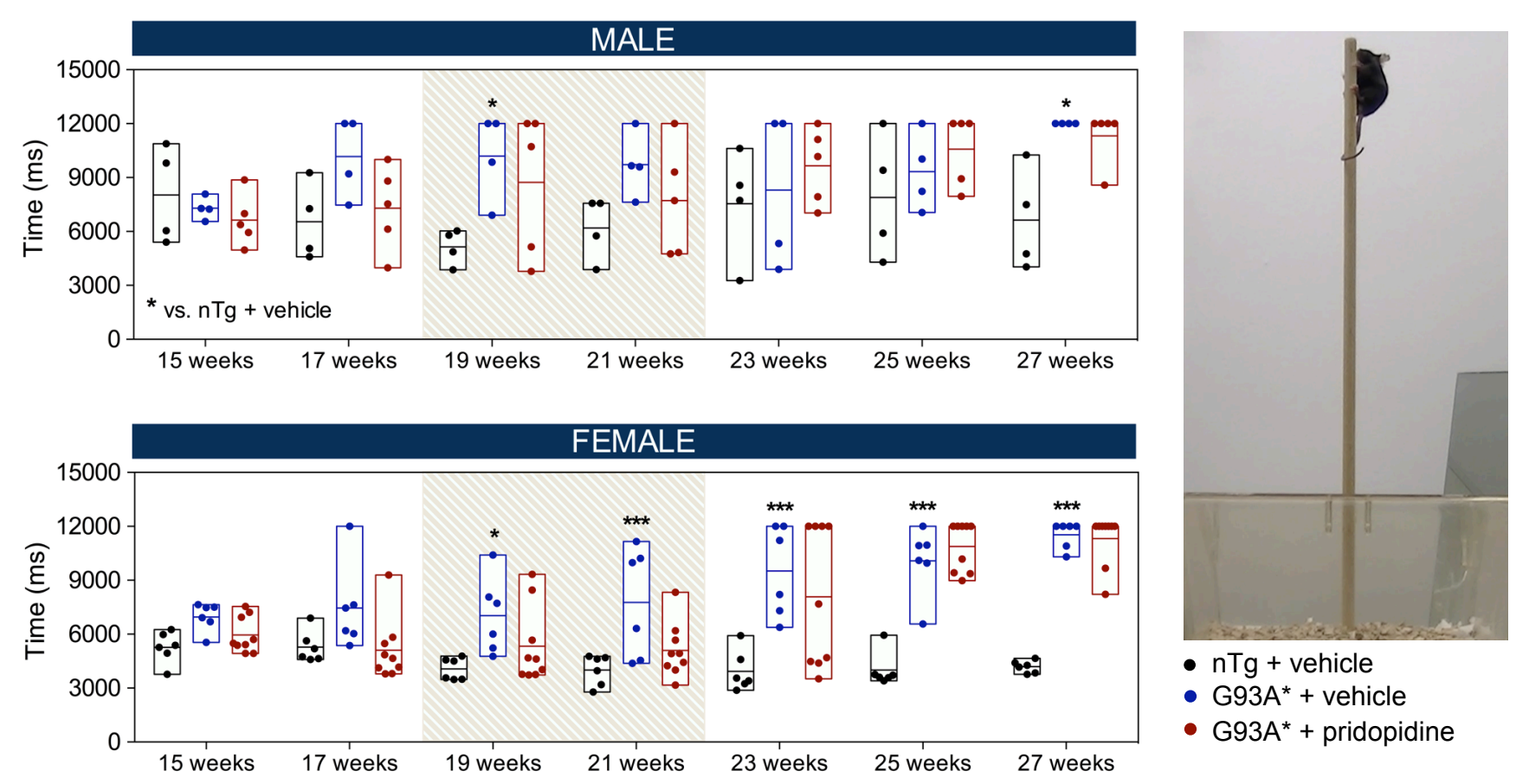

A trend towards improved performance was observed in pridopidinetreated G93A* female mice compared to saline-treated G93A* female mice, but not in males. Data are expressed as mean (min. to max.). ${ }^{*} p<0.05$ and ${ }^{* * *} p<0.001$. Males: $\mathrm{nTg}(\mathrm{n}=4), \mathrm{G} 93 \mathrm{~A}^{*}+$ vehicle $(\mathrm{n}=4)$ and G93A*+pridopidine $(n=5)$. Females: $n T g(n=6)$, G93A*+vehicle $(n=6)$ and G93A* $A^{*}$ pridopidine $(n=9)$.
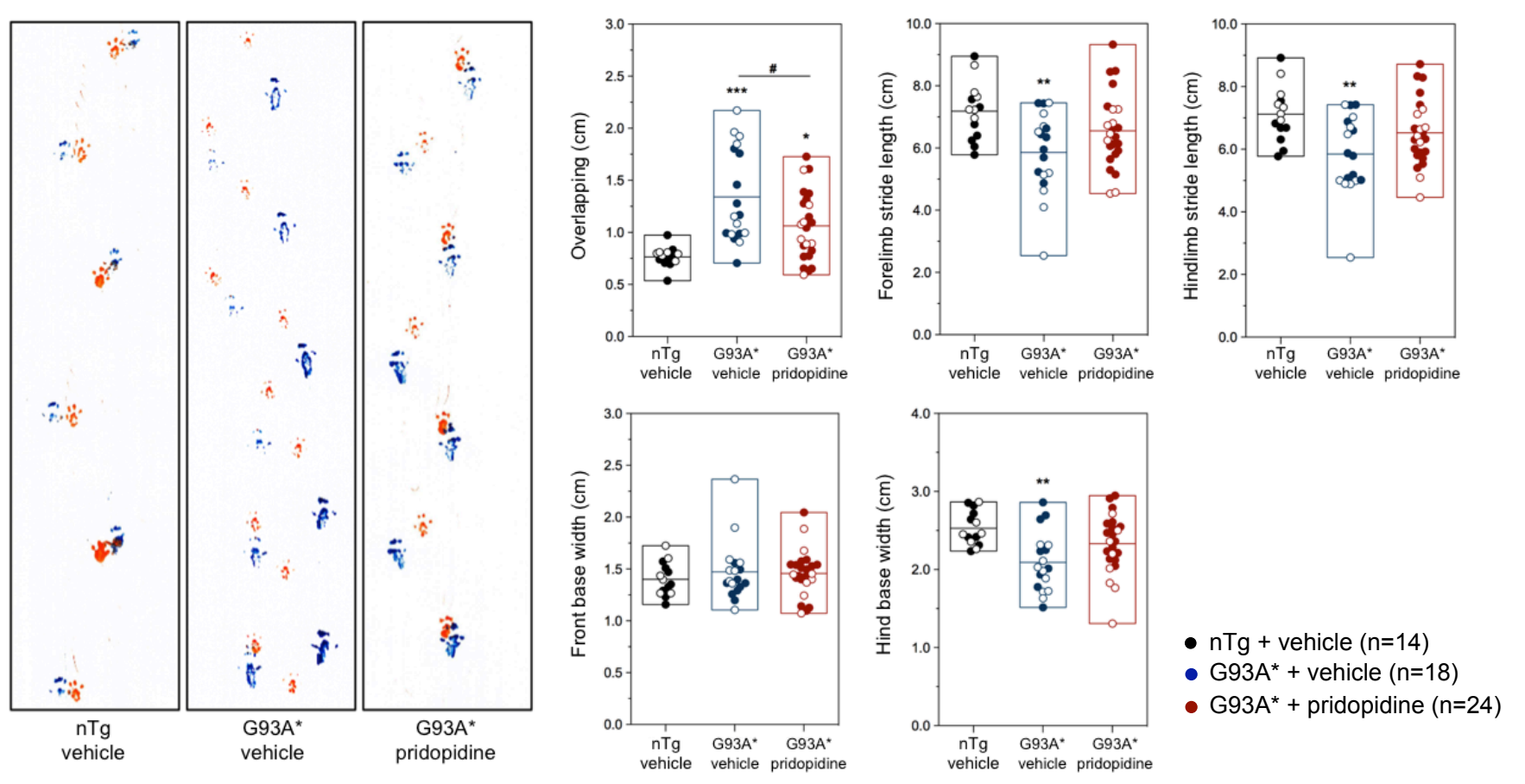

Pridopidine significantly restores gait deficits in G93A* mice. The most representative footprint recordings for each group are presented. Data are expressed as mean (min. to max.). $\left({ }^{*} p<0.05,{ }^{* *} p<0.01\right.$ and ${ }^{* * *} p<0.001$ vs. $\mathrm{nTg} ;{ }^{\#} p<0.05$ vs. G93A* + vehicle).

\section{CONCLUSIONS}

* Pridopidine modified disease progression in which improvements in both motor coordination and life expectancy were observed.

* Some effects of pridopidine were only observed in females.

* The gait test provided clear improvements by pridopidine treatment, 5 weeks after the withdrawal of treatment.

* Transgenic models that exhibit a less aggressive progression of disease are of value to evaluate novel ALS therapies.

\section{ACKNOWLEDGEMENTS}

